\title{
Comparison of a 10 DOF quarter vehicle model with the FE analysis of tire impact against a road obstacle
}

\author{
Nenad Kranjcevic ${ }^{1, a}$, Kristian Gruicic ${ }^{1}$ and Marko Jokic ${ }^{1}$ \\ ${ }^{1}$ Faculty of Mechanical Engineering and Naval Architecture, University of Zagreb, Zagreb, 10002, Croatia
}

\begin{abstract}
In order to investigate the dynamic behaviour of tires on extreme irregularities on the road, a 10 degrees of freedom lumped 2D quarter vehicle model, comprising the two point rigid ring follower tire model, is presented and implemented in Matlab Simulink. The simulation results of tire impact against a step on the road, at various speeds and step heights, are compared to the results achieved by the FE analysis of the same problem.
\end{abstract}

\section{Introduction}

This paper deals with a relatively fast, yet still fairly accurate simulation of the dynamic behavior of a tire impacting an obstacle. Among various papers [1,2] which gave different approaches to the problem, [3] studied a quarter vehicle with different contact, i.e. follower models: point contact, constant footprint, rigid ring, two point and five point rigid ring followers. Compared to the industrial Flexible ring tire model (Ftire) in Simpack, the two point rigid ring follower model was found to be the most accurate. The idea of the two point follower model originates from [4], where the enveloping model with tandem elliptical cams was introduced and implemented as part of a 3D semi-empirical dynamic model of a tire rolling on uneven, but gradually changing road paths. The approach to the problem presented in [4] was adopted in this work, despite the fact that higher steps are under scope. The equations of motion of the 2D quarter vehicle model with 10 degrees of freedom (DOF) are presented in section 2, while the time consuming, but more accurate, finite element (FE) quarter vehicle model is presented in section 3. Sections 4 and 5 give a comparison of these two models and some conclusions, respectively.

\section{The 2D mechanical model for Matlab Simulink implementation}

The mechanical model for the simulation of dynamic behavior of a tire passing over a curb-like obstacle or pothole on the road consists of two sub-models:

- enveloping model and

- quarter vehicle model with rigid ring tire model.

The enveloping model produces from the real road profile the effective profile which is then used as input to the quarter vehicle model to obtain the dynamic response.

\footnotetext{
${ }^{\mathrm{a}}$ Corresponding author : nenad.kranjcevic@fsb.hr
} 


\subsection{The quarter vehicle model with the rigid ring tire model}

The basis for the quarter vehicle model is the tire rigid ring model which comprises three inertial elements: rim $m_{\text {rim }}$, ring $m_{\mathrm{b}}$ and contact mass $m_{\mathrm{c}}$. The fourth inertial element of the system is the mass of the quarter of the vehicle $m_{\text {car }}$. These inertial elements are connected with lumped (discrete) springs and dampers (Fig. 1). For the purpose of controlling the equations of motion, when the wheel is lifted above the road, an additional spring in the vertical direction is added between the contact patch and the road.

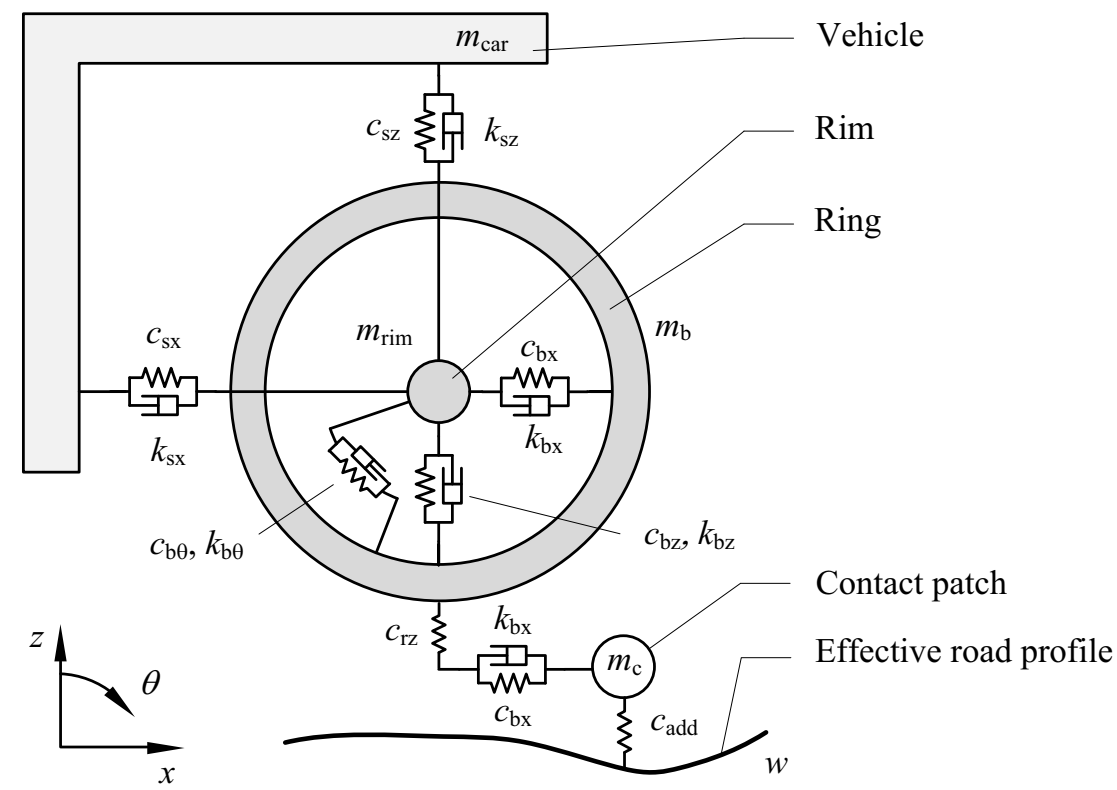

Figure 1. Quarter vehicle model with rigid ring tire model.

The equations of motion of the contact patch can be easily derived:

$$
\begin{aligned}
& m_{\mathrm{c}} \ddot{x}_{\mathrm{c}}=-k_{\mathrm{rx}}\left(\dot{x}_{\mathrm{c}}-\dot{x}_{\mathrm{b}}\right)-c_{\mathrm{rx}}\left(x_{\mathrm{c}}-x_{\mathrm{b}}\right)+C_{\mathrm{c} \kappa} \kappa_{\mathrm{c}} \\
& m_{\mathrm{c}} \ddot{z}_{\mathrm{c}}=-g-c_{\mathrm{rz}}\left(z_{\mathrm{c}}-z_{\mathrm{b}}\right)-c_{\mathrm{add}}\left(z_{\mathrm{c}}-w\right)
\end{aligned}
$$

The last term in Eq. (1) presents the sliding force [4]. Similarly, the equations of motion of the ring are:

$$
\begin{array}{r}
m_{\mathrm{b}} \ddot{x}_{\mathrm{b}}=-k_{\mathrm{bx}}\left(\dot{x}_{\mathrm{b}}-\dot{x}_{\mathrm{rim}}\right)-c_{\mathrm{bx}}\left(x_{\mathrm{b}}-x_{\mathrm{rim}}\right)+k_{\mathrm{bz}} \dot{\theta}_{\mathrm{rim}}\left(z_{\mathrm{b}}-z_{\mathrm{rim}}\right)-k_{\mathrm{rx}}\left(\dot{x}_{\mathrm{b}}-\dot{x}_{\mathrm{c}}\right)-c_{\mathrm{rx}}\left(x_{\mathrm{b}}-x_{\mathrm{c}}\right)+\beta_{\mathrm{y}} F_{\mathrm{cN} 0}, \\
m_{\mathrm{b}} \ddot{\mathrm{z}}_{\mathrm{b}}=-g-k_{\mathrm{bz}}\left(\dot{z}_{\mathrm{b}}-\dot{z}_{\mathrm{rim}}\right)-c_{\mathrm{bz}}\left(z_{\mathrm{b}}-z_{\mathrm{rim}}\right)-k_{\mathrm{bx}} \dot{\theta}_{\mathrm{rim}}\left(x_{\mathrm{b}}-x_{\mathrm{rim}}\right)-c_{\mathrm{rz}}\left(z_{\mathrm{b}}-z_{\mathrm{c}}\right)
\end{array}
$$

and

$$
I_{\mathrm{b}} \ddot{\theta}_{\mathrm{b}}=-k_{\mathrm{b} \theta}\left(\dot{\theta}_{\mathrm{b}}-\dot{\theta}_{\mathrm{rim}}\right)-c_{\mathrm{b} \theta}\left(\theta_{\mathrm{b}}-\theta_{\mathrm{rim}}\right)+r_{\mathrm{e} 0} k_{\mathrm{rx}}\left(\dot{x}_{\mathrm{b}}-\dot{x}_{\mathrm{c}}\right)+r_{\mathrm{e} 0} c_{\mathrm{rx}}\left(x_{\mathrm{b}}-x_{\mathrm{c}}\right)-r_{\mathrm{e} 0} f_{\mathrm{r}} c_{\mathrm{rz}}\left(z_{\mathrm{b}}-z_{\mathrm{c}}\right) .
$$

The last terms in Eq. (3) and (5) are the component of the normal force acting on the ring in horizontal direction and the rolling resistance, respectively. 
The equations of motion of the rim are:

$$
\begin{gathered}
m_{\mathrm{rim}} \ddot{x}_{\mathrm{rim}}=-k_{\mathrm{bx}}\left(\dot{x}_{\mathrm{rim}}-\dot{x}_{\mathrm{b}}\right)-c_{\mathrm{bx}}\left(x_{\mathrm{rim}}-x_{\mathrm{b}}\right)+k_{\mathrm{bz}} \dot{\theta}_{\mathrm{rim}}\left(z_{\mathrm{rim}}-z_{\mathrm{b}}\right)-k_{\mathrm{sx}}\left(\dot{x}_{\mathrm{rim}}-\dot{x}_{\mathrm{car}}\right)-c_{\mathrm{sx}}\left(x_{\mathrm{rim}}-x_{\mathrm{car}}\right), \\
m_{\mathrm{rim}} \ddot{z}_{\mathrm{rim}}=-g-k_{\mathrm{bz}}\left(\dot{z}_{\mathrm{rim}}-\dot{z}_{\mathrm{b}}\right)-c_{\mathrm{bz}}\left(z_{\mathrm{rim}}-z_{\mathrm{b}}\right)-k_{\mathrm{bx}} \dot{\theta}_{\mathrm{rim}}\left(x_{\mathrm{rim}}-x_{\mathrm{b}}\right)-k_{\mathrm{sz}}\left(\dot{z}_{\mathrm{rim}}-\dot{z}_{\mathrm{car}}\right)-c_{\mathrm{sz}}\left(z_{\mathrm{rim}}-z_{\mathrm{car}}\right)
\end{gathered}
$$

and

$$
I_{\text {rim }} \ddot{\theta}_{\text {rim }}=-k_{\mathrm{b} \theta}\left(\dot{\theta}_{\text {rim }}-\dot{\theta}_{\mathrm{b}}\right)-c_{\mathrm{b} \theta}\left(\theta_{\text {rim }}-\theta_{\mathrm{b}}\right)+M_{\text {rim }},
$$

where $M_{\text {rim }}$ is the driving torque. The equations of the vehicle are:

$$
\begin{gathered}
m_{\mathrm{car}} \ddot{x}_{\mathrm{car}}=-k_{\mathrm{sx}}\left(\dot{x}_{\mathrm{car}}-\dot{x}_{\mathrm{rim}}\right)-c_{\mathrm{sx}}\left(x_{\mathrm{car}}-x_{\mathrm{rim}}\right), \\
m_{\mathrm{rim}} \ddot{z}_{\mathrm{car}}=-g-k_{\mathrm{sz}}\left(\dot{z}_{\mathrm{car}}-\dot{z}_{\mathrm{rim}}\right)-c_{\mathrm{sz}}\left(z_{\mathrm{car}}-z_{\mathrm{rim}}\right) .
\end{gathered}
$$

Whenever the wheel is lifted above the road surface, there is no action exerted from the road on the wheel, so the vertical contact force in the additional spring, together with the horizontal component of the normal force and the rolling resistance, i.e. the last terms in Eq. (2), (3) and (5), respectively, must be set to zero.

\subsection{The enveloping tire model - the tandem model with elliptical cams}

The enveloping model and the initial simulation parameters are adopted from [4], together with the additional necessary equations: rolling radius polynomial approximation, approximation of velocity dependent tire sidewall stiffness and sliding force equation, where the $C_{\mathrm{ck}}$ parameter value is adopted from [5].

With non-dimensional parameters $p_{\mathrm{ae}}, p_{\mathrm{be}}, p_{\mathrm{ce}}$ and $p_{\mathrm{ls}}$, which depend on the tire geometry, and the outer radius of an unloaded and un-deformed tire $r_{0}$ according to [4], the ellipse length, height and power, as well as the tandem base length (Fig. 2) are given by:

$$
a_{\mathrm{e}}=p_{\mathrm{ae}} r_{0}, b_{\mathrm{e}}=p_{\mathrm{be}} r_{0}, c=p_{\mathrm{ce}} \text { and } l_{\mathrm{s}}=p_{\mathrm{ls}} 2 a,
$$

respectively.

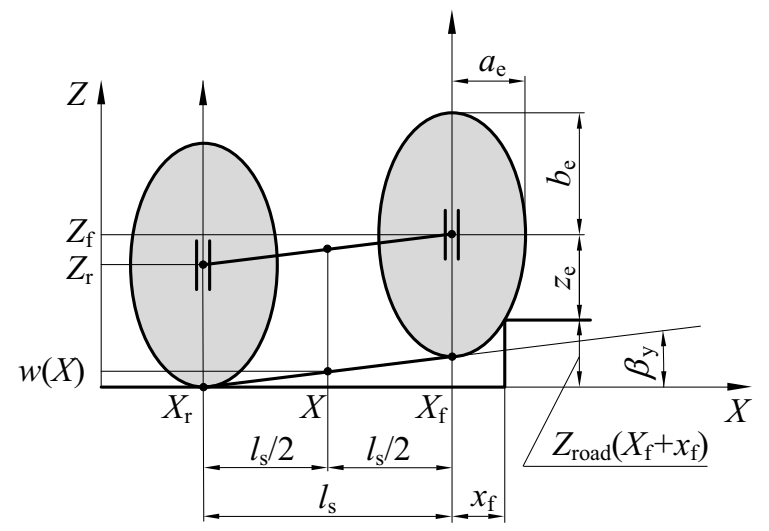

Figure 2. The tandem model with elliptical cams [4]. 
For a given tire and the normal force to the effective road surface

$$
F_{\mathrm{N}}=F_{\mathrm{az}} \cos \beta_{\mathrm{r}} / \cos \left(\beta_{\mathrm{y}}+\beta_{\mathrm{r}}\right),
$$

half of the effective contact patch length $a$ is given by the empirical equation:

$$
a=q_{\mathrm{a} 2} \sqrt{F_{\mathrm{N}}^{2}}+q_{\mathrm{a} 1} \sqrt{F_{\mathrm{N}}}
$$

where the coefficients $q_{\mathrm{a} 1}$ and $q_{\mathrm{a} 2}$ depend on the tire dimensions. Thus, the effective road height is:

$$
w=\left(Z_{\mathrm{f}}+Z_{\mathrm{r}}\right) / 2-b_{\mathrm{e}}
$$

and the effective forward slope of the road:

$$
\beta_{\mathrm{y}}=\operatorname{atan}\left[\left(Z_{\mathrm{r}}-Z_{\mathrm{f}}\right) / l_{\mathrm{s}}\right]
$$

where

$$
Z_{i}=\max \left[Z_{\text {road }}\left(X_{i}+x_{i}\right)+z_{\mathrm{e}}\left(x_{i}\right)\right], \quad i=\mathrm{f}, \mathrm{r}
$$

are center heights of the front and rear elliptical cams, while $z_{\mathrm{e}}$ is the vertical distance from the contact point to the cam center, given by:

$$
z_{\mathrm{e}}=\left|b_{\mathrm{e}}\left(1-\left(\left|x_{\mathrm{f}}\right| / a_{\mathrm{e}}\right)^{c}\right)^{1 / c}\right|
$$

Thus, in the simulation process of rolling the wheel over the road obstacle, the actual road is replaced with the effective road profile, where the enveloping characterics depend on the horizontal position of the wheel center. An example of the enveloping characteristics for a particular tire dimension and road profile is presented in Fig. 3.

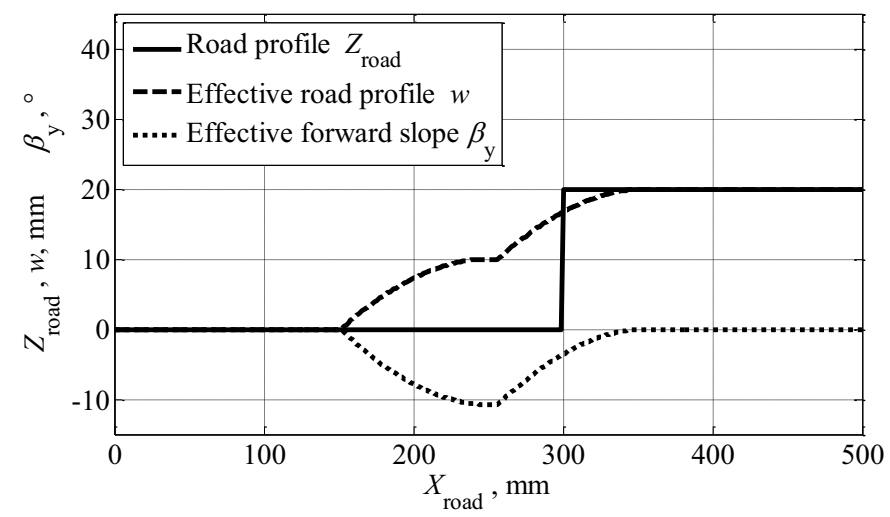

Figure 3. An example of the enveloping characteristics of a 205/60 R15 tire and a road profile with a $20 \mathrm{~mm}$ high step.

\section{The finite element model of the quarter vehicle}

The purpose of the designed FE quarter vehicle model (Fig. 4) is to accurately simulate the tire and obstacle edge impact and establish the resulting quarter vehicle model response. 


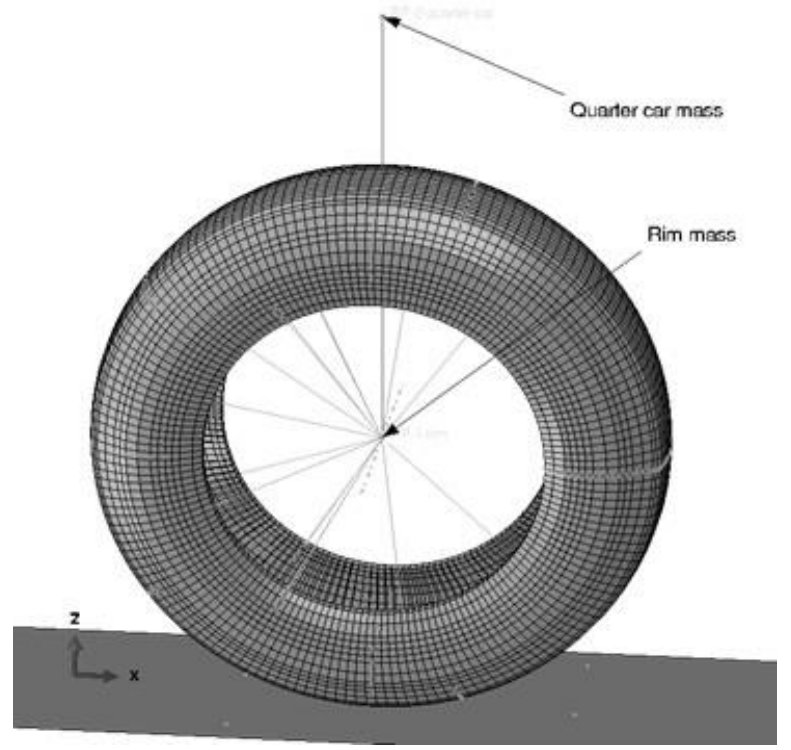

Figure 4. Finite element model.

It comprises the following elements:

- quarter car mass: discrete inertia with mass $240 \mathrm{~kg}$,

- tire (FE model),

- rim mass: discrete inertia located at the rim center, adjusted such that the sum of the total mass of the tire and the rim equals $36 \mathrm{~kg}$,

- spring/dashpot element that connects quarter car mass and rim mass: spring stiffness $16000 \mathrm{~N} / \mathrm{m}$, viscous damping coefficient $980 \mathrm{Ns} / \mathrm{m}$,

- road surface: rigid surface.

The FE model of the tire has been adopted from [6], and the tire dimensions have been adjusted to correspond to the standard 175/65 R14 tire. The tire comprises rubber components (tread, inner liner, sidewall and apex) and reinforcements (carcass and two belts).

The rubber material has been modeled using the 8 node linear continuum elements and the rubber reinforcements are modeled as the embedded rebar elements (i.e. unidirectional fibers). The reinforcements are assumed to be linearly elastic with the modulus of elasticity $172.2 \mathrm{e}+09 \mathrm{~N} / \mathrm{m}^{2}$. The material properties for the rubber material (hyperelastic and viscoelastic properties) are adopted from [4]. The rubber density is $1100 \mathrm{~kg} / \mathrm{m}^{3}$.

\section{Comparison of results}

The simulation was carried out for different initial vehicle speeds $(30,40$ and $50 \mathrm{~km} / \mathrm{h})$ and step heights from 25 to $150 \mathrm{~mm}$. For a particular initial vehicle speed of $40 \mathrm{~km} / \mathrm{h}$ and $150 \mathrm{~mm}$ step height, the rim center displacements for both methods, and for the Simulink model with initial parameter values as in [4], are presented in Fig. 5a. The initial parameter values of the residual stiffness $c_{\mathrm{rz}}$, sidewall stiffness and damping coefficients $c_{\mathrm{bx}}, c_{\mathrm{bz}}, k_{\mathrm{bx}}$ and $k_{\mathrm{bz}}$ in Simulink are then slightly modified to match the FE results in the whole range of initial velocities and step heights. The response obtained using these modified parameters, for the chosen velocity and step height, is presented in Fig. 5b. 
a) Initial parameters $(40 \mathrm{~km} / \mathrm{h}, 150 \mathrm{~mm})$

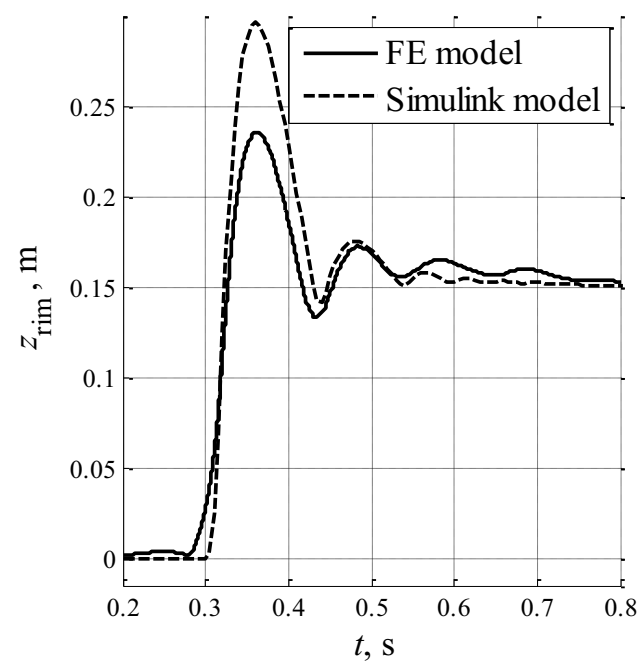

b) Modified parameters $(40 \mathrm{~km} / \mathrm{h}, 150 \mathrm{~mm})$

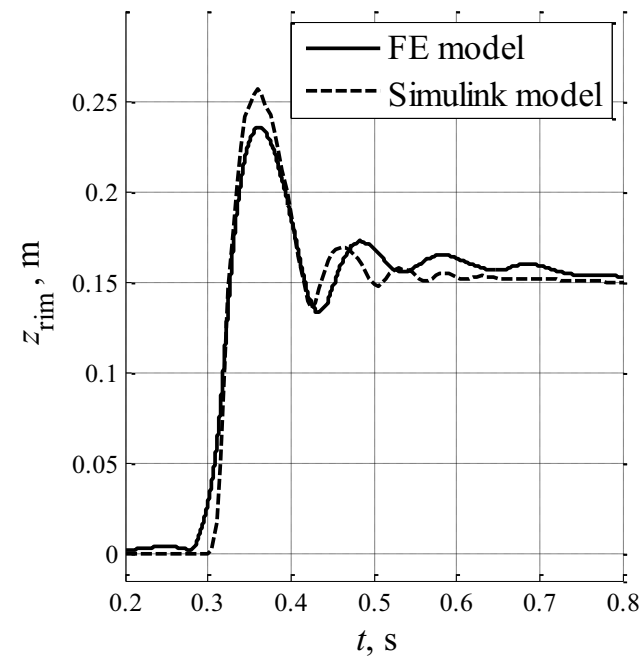

Figure 5. Responses of Simulink model compared to FE model: a) with initial and b) modified Simulink model parameters. Initial vehicle speed is $40 \mathrm{~km} / \mathrm{h}$ and step height $150 \mathrm{~mm}$.

\section{Conclusion}

The presented lumped quarter vehicle with rigid ring tire model gives a significant advantage in calculation speed over the FE simulation (minutes over hours) and fairly accurate results for the amplitude and frequency response during the short period of impact. It could be worth to consider additional optimization of system parameters, which are normally experimentally achieved, to bring the dynamic response of the lumped system even closer to the FE simulation. Both methods remain to be experimentally verified.

\section{Acknowledgments}

The authors gratefully acknowledge the support from Ford Motor Company.

\section{References}

1. A.V.Pesterev, L.A. Bergman, C.A. Tan, J. Sound Vib. 275 127-149, (2004)

2. G.Verros, S. Natsiavas, C. Papadimitriou, J. Vib. Control 11, 581-606, (2005)

3. N.W. Frey, Development of a Rigid Ring Tire Model and Comparison Among Various Tire Models for Ride Comfort Simulations, (Master's thesis, Clemson Univ., 2009).

4. A.J.C. Schmeitz, A Semi-Empirical Three-Dimensional Model of the Pneumatic Tyre Rolling over Arbitrarily Uneven Road Surfaces, (PhD thesis, TU Delft, 2004).

5. M. Schmid, Tire Modeling for Multibody Dynamics Applications, (Thesis, UW-Madison, 2011).

6. C. Wai, O. A. Olatunbosun, J. Terramech. 56, 1-16, (2014). 